\title{
MISCDLANEA
}

\section{METÁFORAS SOBRE MODELOS EDUCATIVOS EN EDUCACIÓN PARA LA SALUD: UNA PROPUESTA DE AULA DE SALUT.}

\author{
Salvador Sáez ${ }^{a}$ \\ Ester Arimon ${ }^{b}$ \\ Laia Sióc \\ CarmenTorres Penella ${ }^{d}$ \\ Miguela Martínez \\ Roser Palau Costafreda ${ }^{f}$
}

a: Departamento de Enfermería. Universidad de Lleida.

b: Clínia del Pilar-Sant Jordi. Barcelona.

c: Clínica de Montserrat. Lleida.

d: Departamento de Enfermería. Universidad de Lleida.

e: Centro de Atención Primaria Baláfia. Institut Català de la Salut. Lleida.

f: Hospital Mútua de Tarrassa. Barcelona.

a, b, c, d, e, f: Departament d'Infermeria Universitat de Lleida (UdL), miembros de "Aula de Salut".

E-mail: ssaez@infermeria.udl.cat 


\section{RESUMEN}

\section{Título:}

Metáforas sobre modelos educativos en Educación para la Salud: una propuesta de Aula de Salut.

Las metáforas son un instrumento pedagógico y terapéutico para facilitar la comunicación y comprensión de temas relacionados con la salud-enfermedad.

\section{Objetivo:}

Presentar las metáforas que el equipo de "Aula de Salut" ha elaborado en base a la experiencia en formación de grado, posgrado y profesionales, intervención y asesoramiento en Educación para la Salud (EpS), con el fin de reflexionar y enseñar los modelos de intervención en EpS.

\section{Método:}

Se ha seguido la metodología de la investigación-acción de Mac Kernan.

\section{Resultados:}

Se han caracterizado cuatro tipologías distintas de profesionales de la salud en función de su modelo de intervención (botijo, maceta, látigo y matrona) con sus respectivas metáforas. A través de dichas metáforas se provoca la implicación, tanto del alumno como del profesional, permitiéndoles observar y analizar sus modelos de intervención para ayudarles a comprenderlos y mejorarlos.

\section{Conclusiones:}

Ha resultado ser una herramienta muy útil porque abre una vía de investigación en el campo de la EpS tanto en la práctica asistencial como en la formación de los profesionales de la salud. También constituye un elemento clave de comunicación al convertirse en un modo de transmitir el mensaje de forma más efectiva $y$, finalmente, se alzan como una herramienta de pensamiento al transformarse en un medio para ayudar a entender nuevas ideas, conceptos y métodos.

Palabras clave: Formación. Educación para la Salud. Metáforas. 


\section{ABSTRACT}

\section{Title:}

Metaphors as an educational model for health education: a proposal from "Aula de Salut".

Metaphors are a pedagogical and therapeutic tool to facilitate communication and understanding of issues related to health and disease. Objetive:

To present the metaphors that the team of "Aula de Salut" has been developed based on experience in teaching graduates, post-graduates and professionals, intervention and counseling in Health Education (HE), to think and teach intervention models in

\section{Methods:}

Has followed the methodology of action research Mac Kernan. Results:

Has characterized four types of health professionals based on their model of intervention (jug, pot, whip and a midwife) with their metaphors. The use of the aforementioned metaphors are causes of the involving both students and professionals, allowing them to observe and analyze models of intervention to help them understand and improve outcomes.

\section{Conclusions:}

It has proved a very useful tool because it presents a means for researching in the field of $\mathrm{HE}$ both in terms of practical assistance and in training of health professionals. It is also a key element facilitating more effective communicationa and, finally, it presents as a way of thinking it becomes a vehicle for understanding new ideas, concepts and methods.

Key words: Training, Health education, Metaphors. 


\section{INTRODUCCIÓN}

En las intervenciones educativas-asistenciales con los usuarios y familias se usan las metáforas como instrumento pedagógico y terapéutico para facilitar la comunicación y comprensión de temas relacionados con la saludenfermedad. Su valor ha sido validado por la comunidad científica1,2,3. Así, para Lakoff y Johnson ${ }^{3}$, "La esencia de la metáfora es entender y experimentar un tipo de cosas en términos de otra". Han demostrado que nuestros sistemas conceptuales están constituidos para operar metafóricamente; en este sentido, la mayoría de conceptos especialmente aquellos que son abstractos o complejos, son entendidos, como mínimo, en parte en términos de otros conceptos más familiares. Para estos autores, la metáfora "impregna la vida cotidiana, no solamente el lenguaje, sino el pensamiento y la acción".

Lo atractivo de la metáfora, dicen Costa y López 4 "es que resulta un sutil juego al comunicar una cosa en términos de otra, además de permitir con variables escenas y personajes que suscitan y despiertan emociones susceptibles de ser bien percibidas y aceptadas desde diferentes perspectivas".

Insisten estos autores que la metáfora es también "un recurso de gran sutileza para comunicar mensajes y contenidos difíciles de aceptar, así como incitar a la experimentación y al cambio de perspectiva sin provocar resistencias" por eso también su utilidad en los procesos de formación de futuros profesionales.

Sanjurjo y Rodríguez ${ }^{5}$ defienden el uso pedagógico y didáctico de las metáforas como: a) un amplificador cognitivo que permite aumentar las posibilidades de contracción y apropiación de nuevos conocimientos; b) valor heurístico, ya que permite generar y organizar nuestro conocimiento acerca de la realidad; c) para enriquecer la propia idea que se pretende transmitir; d) para generar nuevas descripciones de un fenómeno menos conocido en términos de otro más conocido.

Salleras ${ }^{6}$ ya habló de diferentes etapas en las que coinciden modelos de 
intervención en función del rol del paciente y profesional y la importancia del contexto. Con otras taxonomías y nomenclaturas otros autores 7,8,9,10 han presentado sus modelos educativos. Coinciden a manera de síntesis en los enfoques transmisores o reproductores, otros más prácticos y contextuales y otros más críticos o transformadores.

Desde la experiencia en formación a los alumnos de grado, postgrado y profesionales, intervención y asesoramiento en Educación para la Salud (EpS), el equipo de "Aula de Salut", propone sus metáforas como una propuesta didáctica para reflexionar y enseñar los modelos de intervención en EpS al alumnado de grado y postgrado y profesionales de salud. Con ello, se caracterizan cuatro tipologías distintas de profesionales de la salud en función de su modelo de intervención en EpS.

\section{Objetivos:}

Con esta propuesta se pretende:

1. Presentar y analizar los modelos educativos de los profesionales de la salud derivados de las intervenciones en EpS y desde el marco metafórico.

2. Describir las ventajas de conocer y reflexionar sobre nuestras intervenciones educativas-asistenciales con estas metáforas por parte de los profesionales y alumnado.

3. Poder identificar y trabajar con metáforas como otro recurso pedagógico en EpS.

\section{MÉTODO}

El estudio se ha llevado a cabo siguiendo el proceso y la metodología de la investigación-acción de MacKernan ${ }^{11}$ citado por Álvarez-Gayou ${ }^{12}$. El propósito de la misma es resolver problemas cotidianos e inmediatos. Está centrado en la búsqueda de mejores resultados, ayudado por la participación de los actores, quienes al mismo tiempo aprenden y se desarrollan como 
personas.

La experiencia se ha venido realizando en contextos formativos. Durante varios años se ha trabajado los posibles modelos de intervención educativaasistencial y sus características con el alumnado del grado de Enfermería, los profesionales en talleres y cursos de formación en EpS y con el alumnado de los masteres oficiales en Ciencias de la Enfermería y en Educación para la Salud.

Usamos en nuestra experiencia metáforas para enseñar y analizar modelos educativos en los diferentes cursos y asignaturas con los participantes y evaluamos el potencial de usarlas como estrategia de instrucción. También exploramos y valoramos el hecho de trabajar con metáforas en salud para su posterior implicación en intervenciones educativas-asistenciales.

En función de las variables analizadas: rol del usuario, rol del profesional, modelo educativo, objetivo de la intervención y tipo de comunicación y relación, se proponen cuatro modelos educativos con sus respectivas metáforas, que a la vez caracterizan cuatro tipologías distintas de profesionales de la salud en función de su modelo de intervención en EpS.

\section{RESULTADOS}

Fruto del análisis e interpretación de la bibliografía consultada respecto a modelos educativos en EpS así como del feedback con la experiencia en la implementación docente, se proponen cuatro modelos educativos con sus respectivas metáforas: botijo, maceta, látigo y matrona. En el cuadro de síntesis, se presentan sus características (correspondientes a cuatro tipologías de profesionales de la salud en función del modelo de intervención) y la iconografía de soporte para reforzarlas. (Tabla 1. Metáforas para analizar e interpretar los modelos de intervención en EpS). 


\section{MODELO "BOTIJO"}

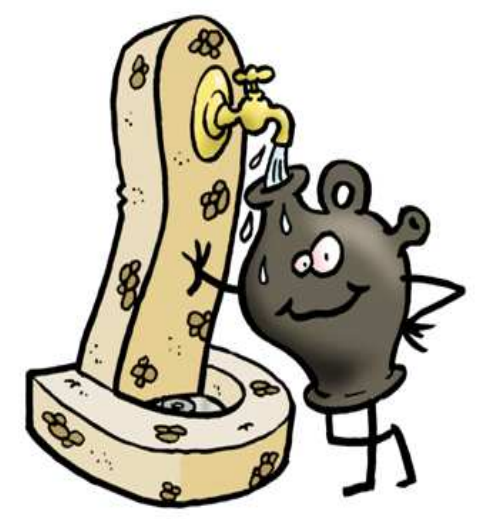

Figura 1. Modelo Botijo.

El modelo ideológico que sigue el profesional de la salud tipo botijo es el modelo tradicional. La medicina occidental ha entendido históricamente al usuario como un ser bastante pasivo y negativo en relación con el cuidado de su salud. Por consiguiente, hay que guiarle y obligarle a que se dirija hacia las metas que constituirán su bienestar. No hay que contar con los deseos del usuario, porque se presupone que éste no sabe qué es lo mejor para él.

El modelo que sigue el profesional tipo botijo es un modelo cultural, es decir, centrado en el contenido. El objetivo de la intervención profesional es la pura transmisión de conocimientos. Si el profesional de la salud redactara objetivos de forma honesta, tendrían la estructura "que yo haga...", "que yo, que soy el profesional, transmita los conocimientos adecuados acerca de...". El contenido de los objetivos es la enfermedad y la curación.

El profesional de la salud tipo botijo tiene una concepción vertical y autoritaria del proceso de comunicación con el usuario de la salud. Este profesional es rutinario y paternalista en su trabajo, que ejerce desde la autoridad. El profesional de la salud se encuentra en el epicentro del proceso de comunicación. Su misión como comunicador es transmitir conocimientos. 
Los conocimientos se depositan donde el profesional considera que hace falta. Una buena parte de su trabajo es dar pequeñas charlas magistrales al usuario de la salud. Este profesional dicta normas y reglas, y prescribe sin consultar la opinión del usuario. El profesional de la salud es el que sabe y tiene un concepto unidireccional, y no recíproco, de la transmisión del saber.

Frente al profesional tipo botijo, al usuario de la salud no le queda más remedio que actuar de simple recipiente vacío o "contenedores vacíos" según Max van Manen ${ }^{13}$. El profesional va echando los conocimientos y saberes - lo que sería el agua - dentro del usuario, a quien el profesional ignora. El profesional actúa bajo el supuesto de que el usuario no tiene ni experiencia previa, ni creencias, ni conocimientos acerca de la salud.

El profesional que sigue este modelo educativo presupone que el usuario le obedecerá por el simple hecho de que él posee la autoridad intelectual. El diálogo y la participación no existen. Nos encontramos con lo que Paulo Freire llama "concepción bancaria" de la educación ${ }^{14}$.

En el ámbito familiar, el profesional tipo botijo ofrece información y sugerencias sanitarias, pero no desea interactuar con ellas más allá de lo estrictamente profesional. Normalmente, lo hace para ahorrar tiempo, porque los familiares suelen formular más preguntas que los usuarios.

El profesional tipo botijo favorece, con esta superioridad intelectual, una respuesta interior a menudo agresiva en el usuario, el cual, para no ponerla de manifiesto se muestra poco activo, con dificultades de comunicación y poca confianza en la cooperación. Cuando se impide el feedback, al usuario se le dificulta la captación del mensaje emitido por el profesional de la salud. Ello engendra apatía, inseguridad y miedo a equivocarse al llevar a cabo la prescripción. En muchas ocasiones, el miedo provoca incumplimiento terapéutico. El usuario prefiere no hacer nada a hacer algo mal. El usuario 
toma una actitud pasiva, de mero recipiente.

En caso de que el conflicto se evidencie, el profesional de la salud tipo botijo no tiene en cuenta el conflicto, lo desprecia. En la consulta, el diálogo y la participación no existen, porque, por su forma de entender el ejercicio de su profesión, si se "entretuviera charlando", no acabaría a la hora.

Todavía es muy frecuente encontrar profesionales de la salud que son los únicos que hablan cuando se encuentran con usuarios. El profesional tipo botijo abunda en las consultas externas de los hospitales y se puede encontrar también en atención primaria.

La perspectiva es siempre jerárquica, unidireccional, del que sabe al que no sabe. El método de aprendizaje es secundario.

\section{MODELO "MACETA"}

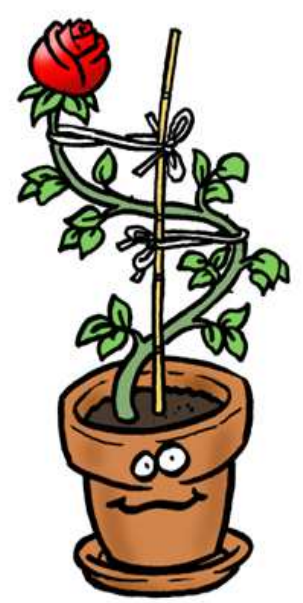

Figura 2. Modelo Maceta.

El profesional de la salud tipo maceta tiene una concepción familiar de la intervención sanitaria. De hecho, cree que en la base de la intervención se encuentran el individuo, la familia y el equipo sanitario. Entiende que la familia 
influye sobre la salud de sus miembros en un sentido positivo, como recurso fundamental para colaborar en el cuidado y resolución de problemas, y en un sentido negativo, como generadora de enfermedad, hábitos de vida insanos y conductas poco saludables. El profesional de la salud tipo maceta se esfuerza por comprender el contexto de la enfermedad. Sin embargo, no suele captar la dimensión comunitaria de la atención profesional. El individualismo personal e institucional en el que trabaja, dificulta tanto la concepción como la intervención comunitaria, que deben hacerse sobre la base de una coordinación intersectorial.

El profesional de la salud tipo maceta es el que determina qué maceta, qué semillas, qué abono hay que utilizar... La planta es la que ha de crecer, pero es el profesional quien guía el crecimiento. El profesional programa la planta, utilizando todas las metodologías que conoce: los trípticos, los audiovisuales, etc. Si el profesional cree que hay que podar la planta, lo hace por su bien. En el fondo, la actitud del profesional tipo maceta es autoritaria, como en el modelo anterior, pero sus formas son muy distintas. El profesional de la salud es simpático, amable y cordial. Deja opinar y discutir las decisiones, pero siempre acaba imponiendo su parecer.

El modelo que sigue el profesional de la salud tipo maceta es de tipo social, es decir, está centrado en el equipo de profesionales y en el grupo de referencia del usuario. El objetivo de la intervención profesional es la construcción de conocimientos y comportamientos. El profesional de la salud cree en el poder de los objetivos, que tienen la forma "que se haga...", "que el equipo de profesionales consiga...". El contenido de los objetivos es el tratamiento de problemas específicos de salud.

Este profesional permite que haya una cierta bidireccionalidad en el proceso de comunicación con el usuario. El profesional de la salud cree que su misión es influir en el usuario de la mejor manera posible. Una buena parte de 
su trabajo es de empatía. El profesional actúa bajo el supuesto de que si conecta emocionalmente con el usuario, éste le obedecerá por el simple hecho de que tiene la autoridad moral.

En este modelo, el usuario de la salud parece estar en construcción. El usuario tiene experiencia y conocimientos previos, pero se encuentran "a medio montar". El profesional de la salud es el que sabe dónde están los errores y cómo corregirlos. En algunos casos, el profesional tipo maceta deja que los usuarios lleven sus actitudes hacia comportamientos poco saludables, por una apariencia de respeto a la iniciativa personal. Entonces, los usuarios se sienten descontentos con la actuación del profesional.

En relación con la familia, este profesional ofrece apoyo emocional. Suele hacer preguntas que posibiliten que los miembros de la familia expresen sus preocupaciones y emociones relacionadas con la situación del enfermo y su repercusión en ellos mismos y les escucha empáticamente.

Suele preocuparse por la imagen que da al usuario. Por este motivo, para él la posibilidad de un enfrentamiento es un grave problema. Las relaciones entre el profesional de la salud tipo maceta y el usuario suelen ser ambivalentes. Se trata de una relación necesaria, pero al mismo tiempo temida. En caso de que aparezca un conflicto, éste se habla en las reuniones de equipo. En la consulta, el conflicto se ignora hasta que no se haya encontrado una solución, porque el usuario no puede dar una opinión profesional.

Actualmente, es frecuente encontrar profesionales de la salud cuyo objetivo principal es hacerse amigos del usuario. El profesional tipo maceta se encuentra con facilidad en la atención primaria, si bien una parte de los profesionales de la atención hospitalaria hace esfuerzos por aproximarse al modelo. 


\section{MODELO "LÁTIGO"}

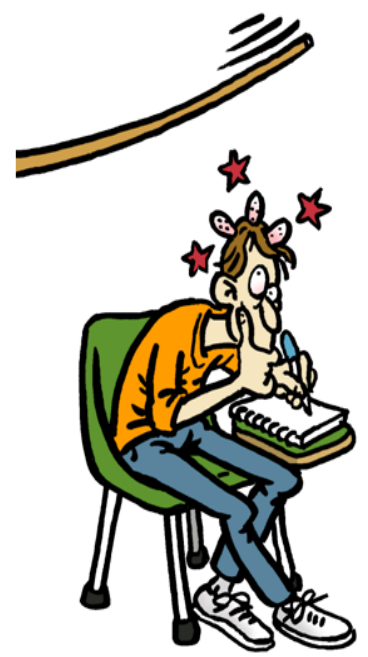

Figura 3. Modelo Látigo.

El modelo ideológico que subyace al profesional de la salud tipo látigo tiene una base conductista. El profesional actúa bajo el supuesto de que el problema de salud que presenta el usuario es consecuencia de su mal comportamiento, de sus malos hábitos: conducir demasiado deprisa, beber alcohol, tener relaciones sexuales sin protección... El profesional tipo látigo cree que amenaza al usuario por su bien y por lo tanto, éste le obedecerá porque el profesional tiene la autoridad social.

Al profesional de la salud tipo látigo le gusta tomar todas las decisiones. No suele dar explicaciones de su actuación. El profesional toma las sugerencias y opiniones de los usuarios como críticas a su actuación profesional y en esas ocasiones llegan a emplear la censura y las amenazas. Tiene una actitud agresiva cuando alguien se resiste a su voluntad. Su principio es que "la letra, con sangre entra".

El modelo que sigue el profesional tipo látigo es de tipo cultural, es decir, está centrado en la actividad. El objetivo de la intervención profesional es la modificación de conducta. Si el profesional de la salud redactara objetivos de forma honesta, empezarían con "que yo le haga hacer...", "que yo, que soy el 
profesional, consiga que el usuario se comporte de manera adecuada acerca de...". El contenido de los objetivos se centra en el cuidado esporádico de los trastornos de la salud.

El profesional de la salud tipo látigo tiene una concepción vertical y autoritaria del proceso de comunicación con el usuario de la salud, igual que el profesional tipo botijo, pero no paternalista. Su misión es conseguir un cambio de comportamiento determinado. Los medios para conseguir el fin son las amenazas (látigos): "si no comes verdura...", "si no adelgazas veinte kilos, no hace falta que vuelvas por la consulta...". El profesional de la salud tipo látigo también se encuentra en el epicentro del proceso de comunicación, pero en la consulta la base de la actuación profesional es el castigo por el error del usuario.

Frente al profesional tipo látigo, el usuario de la salud se siente muy dirigido. En este caso, el profesional no ignora al usuario, sino que le ataca de manera psicológica con su "ordeno y mando". O como muy bien expresa Philippe Meirieu: "sin aceptar su no-poder"15.

Cuando se encuentran con profesionales tipo látigo, muchos usuarios acumulan una hostilidad y un descontentamiento que puede estallar en cualquier momento. No obstante, en caso de que aparezca un conflicto, el profesional lo martillea. En la consulta, el profesional tipo látigo ostenta la verdad frente al pobre usuario, que va equivocado por la vida.

Este tipo de profesional es fácil de encontrar tanto en la atención hospitalaria como en la primaria, y más frecuentemente cuanto mayor sea la ratio de pacientes por profesionales. 


\section{MODELO "MATRONA"}

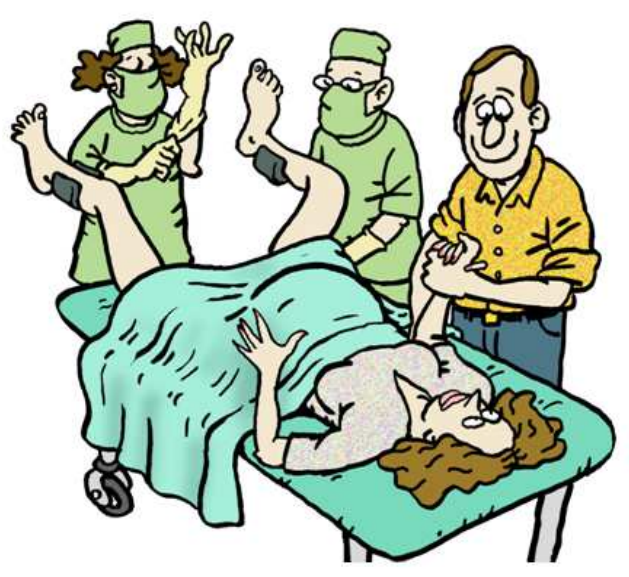

Figura 5. Modelo Matrona.

El profesional de la salud tipo matrona reconoce el valor de los conocimientos y las experiencias de los demás y se halla dispuesto a cooperar con ellos y a adaptarse a sus objetivos. El profesional también persigue un cambio de actitudes y de comportamiento, como el tipo látigo, pero a partir de un ciclo compuesto por la acción, la participación, la crítica, y la reflexión. El profesional de la salud tipo matrona cree que nadie se educa sólo, que nadie se hace a sí mismo. Todos aprendemos de los demás. El profesional parte del principio "aprender a aprender".

El profesional de la salud tipo matrona intenta adaptarse en cada momento a las necesidades y a las características del usuario. Intenta que su intervención sea solamente la de catalizador y de promotor de las decisiones de éste, para que asuma la responsabilidad de ser consecuente con las decisiones tomadas.

El modelo que sigue el profesional de la salud tipo matrona es de tipo educativo, es decir, está centrado en la persona. El objetivo de la intervención profesional es el desarrollo personal. El profesional de la salud no se plantea establecer objetivos independientemente de los usuarios. En este caso, los objetivos tienen la forma: "que todos nosotros hagamos..." o, si se da el caso, "que los participantes consigan...". El contenido de los objetivos se traslada del 
tratamiento de la enfermedad a la promoción de la salud, la prevención de los problemas y el cuidado global de las personas.

El profesional de la salud tipo matrona intenta que haya la máxima interacción en el proceso de comunicación con el usuario de la salud. Entiende la intervención profesional como un proceso dentro de un contexto que nos marca continuamente. Antes de hacer una recomendación sanitaria, el profesional consulta la opinión del usuario, acepta sus sugerencias y comparte con él la responsabilidad. La actitud del profesional de la salud tipo matrona es una actitud de ayuda, de comprensión y de aceptación del usuario, dándole ánimos y ayudándole a clarificar sus problemas.

Frente al profesional de la salud tipo matrona, el usuario no tiene la sensación de encontrarse solo, ni dirigido, ni vacío. Siempre se encuentra con, al menos, un grupo de referencia. Se crea una relación pedagógica. "No importa para que pero yo estoy aquí. Y puedes contar conmigo" ${ }^{13}$.

En el ámbito familiar, el profesional de la salud tipo matrona reconoce su propia participación en el sistema. A partir de sus conocimientos, trabaja con un enfoque sistémico e intervenciones planificadas. Entiende que la dinámica interna personal y del grupo de referencia es mayor cuanto menos dominante sea el profesional de la salud.

En caso de que aparezca un conflicto, el profesional de la salud tipo matrona lo considera como una ocasión para el aprendizaje. En la consulta, el error se acepta como elemento necesario dentro de todo proceso.

El profesional de la salud tipo matrona puede encontrarse en aquellos ámbitos laborales privilegiados en los que el nivel de formación en atención comunitaria sea el adecuado y la presión asistencial no sea tan fuerte como es habitual. Entonces, los profesionales pueden practicar el cambio de paradigma. 


\section{DISCUSIÓN}

Las metáforas son un gran recurso didáctico y terapéutico para incitar a la experimentación y al cambio de perspectivas sin provocar resistencias.

Coincidimos con Costa y López ${ }^{4}$ cuando afirman que la metáfora "es un enorme recurso didáctico porque concita una gran riqueza de significados y de connotaciones emocionales, que hacen que lo que expresemos se comprende y se retenga mejor y además tenga mayor impacto".

Estamos en sintonía con Cook y Gordon ${ }^{16}$ cuando afirman que las metáforas ayudan a los alumnos a crear eslabones creativos e imaginativos entre los conceptos existentes y los asociados a los nuevos conocimientos, facilitando su asimilación y comprensión. Se crea con ello también todo un campo para la investigación y práctica educativa.

De acuerdo con Sapory ${ }^{17}$ cuando presenta el análisis de metáforas para ser también utilizados en procesos formativos para evaluar modelos.

En EpS la metáfora es un recurso excelente ya que permite configurar un mensaje de una manera inusual, atractiva y llena de matices, como opinan también Costa y López 4 .

El uso didáctico de las metáforas nos permite concretar conocimientos abstractos y ayudar al establecimiento de relaciones entre los nuevos y viejos. Trabajar con metáforas ofrece un enorme potencial para contribuir al aprendizaje a partir de lo ya conocido, y para relacionar, organizar y trasformar el nuevo conocimiento. Tal como dice Mariano Martín Gordillo ${ }^{18}$, "es evidente que la reflexividad que supone aplicar muchos conceptos científicos de modo metafórico a la realidad de las actividades de enseñanza y aprendizaje en las 
aulas tiene una enorme potencia para desvelar muchos aspectos de la vida escolar. El desgaste al que se ven sometidos los docentes innovadores en la mayoría de las instituciones escolares frente al predominio de los conservadores que, casi sin esfuerzo, consiguen destruir lo que aquéllos trabajosamente han construido, puede quedar muy bien reflejado y hasta explicado".

De acuerdo con la escritora Susan Sontag: "Es imposible residir en el reino de los enfermos sin dejarse influenciar por las siniestras metáforas con que han pintando su paisaje" 19 .

\section{CONCLUSIÓN}

El proceso de enseñanza-aprendizaje mediante metáforas, provoca la implicación del alumno y del profesional, permitiéndoles observar y analizar sus modelos de intervención y de esta manera, ayudarles a comprenderlos y mejorarlos.

Los profesionales de la salud dedicados a conseguir cambios de comportamientos saludables deberían dominar su uso. Por este motivo, está justificado que se enseñe en el aula y promueva la investigación sobre las mismas.

La explicación y uso de dicho material en la formación de grado y posgrado ha permitido facilitar el debate y el análisis de los modelos en EpS y observar que las supuestas visiones deformadas de los modelos de EpS son, en realidad, visiones conformadas históricamente.

Podemos concluir que, por un lado, nos encontramos ante una herramienta muy útil como: 
a) estrategia para la enseñanza y aprendizaje, y abre una vía para su investigación en el campo de la EpS, tanto en la práctica asistencia como en la formación de los profesionales de la salud.

b) elemento clave de comunicación. Constituye un modo de trasmitir el mensaje de manera más efectiva.

c) y como herramienta de pensamiento. Un medio para ayudar a entender nuevas ideas, concepto, métodos...

Y por otro lado, comprobamos que las metáforas afectan a la manera en que percibimos, pensamos y actuamos y ayudan a los alumnos y profesionales a pensar metafóricamente.

Tabla 1: Metáforas para analizar e interpretar los modelos de intervención en EpS.

\begin{tabular}{|c|c|c|c|c|}
\hline METÁFORAS & BOTIJO & MACETA & LATIGO & MATRONA \\
\hline ROL USUARIO & $\begin{array}{l}\text { Pasivo } \\
\text { "recipiente } \\
\text { vacío" }\end{array}$ & Activo & $\begin{array}{l}\text { "Responsables de } \\
\text { sus hábitos" }\end{array}$ & $\begin{array}{l}\text { Activo } \\
\text { Participativo }\end{array}$ \\
\hline ROL PROFESIONAL & $\begin{array}{l}\text { Guiarle } \\
\text { Obligarle }\end{array}$ & $\begin{array}{l}\text { Tutor } \\
\text { Paternalista }\end{array}$ & $\begin{array}{l}\text { Autoritario } \\
\text { Amenazar } \\
\text { Marcar }\end{array}$ & $\begin{array}{l}\text { Cooperación } \\
\text { Catalizador }\end{array}$ \\
\hline $\begin{array}{c}\text { MODELO } \\
\text { EDUCATIVO }\end{array}$ & $\begin{array}{l}\text { Centrado en el } \\
\text { contenido }\end{array}$ & $\begin{array}{l}\text { Centrado en el } \\
\text { contexto y equipo }\end{array}$ & $\begin{array}{l}\text { Centrado en la } \\
\text { actividad }\end{array}$ & $\begin{array}{l}\text { Centrado en la } \\
\text { persona }\end{array}$ \\
\hline $\begin{array}{l}\text { OBJETIVOS DE LA } \\
\text { INTERVENCION }\end{array}$ & $\begin{array}{l}\text { Transmisión de } \\
\text { conocimientos }\end{array}$ & $\begin{array}{l}\text { Construir } \\
\text { conocimiento y } \\
\text { comportamientos }\end{array}$ & $\begin{array}{l}\text { Modificar } \\
\text { conductas }\end{array}$ & $\begin{array}{l}\text { Desarrollo } \\
\text { personal }\end{array}$ \\
\hline $\begin{array}{c}\text { TIPO DE } \\
\text { COMUNICACIÓN Y } \\
\text { RELACION }\end{array}$ & $\begin{array}{l}\text { Vertical } \\
\text { Unidireccional }\end{array}$ & $\begin{array}{l}\text { Horizontal } \\
\text { Bidireccional }\end{array}$ & $\begin{array}{l}\text { Vertical } \\
\text { Unidireccional }\end{array}$ & $\begin{array}{l}\text { Bidireccional } \\
\text { Interrelación }\end{array}$ \\
\hline
\end{tabular}




\section{BIBLIOGRAFÍA}

1. Bustos, E. La metáfora. Ensayos transdisciplinares. Madrid: FCE/UNED; 2000.

2. Gwyn, R. Comunicating Health and Illnes. London: SAGE Publications, 2002.

3. Lakoff, G.; Johnson, JL. Metáforas de la vida cotidiana. Madrid: Cátedra; 2001.

4. Costa, M., López, E. Educación para la Salud. Guía práctica para promover estilos de vida saludables. Madrid: Pirámide; 2008.

5. Sanjurjo, L; Rodríguez, X. Volver a pensar la clase. Las formas básicas de enseñar. Argentina: Homo Sapiens; 2003.

6. Salleres, L. Educación Sanitaria. Principios, métodos y aplicaciones. Madrid: Díaz de Santos; 1990.

7. Flores, M.D. Educación para la salud y la Enfermería. Murcia: Diego Marín LIBRERO EDITOR; 2001.

8. Mazarrasa, M. y col. Salud Pública y Enfermería Comunitaria. Madrid: McGRAW-HILL- INTERAMERICANA; 1996.

9. García, A.; Sáez, J.; Escarbajal, A. Educación para la salud. Madrid: Arán; 2000.

10.Polaino-Lorente, A. Educación para la salud. Barcelona: Herder; 1987.

11.MacKernan Jamés, Investigación-acción y currículo, Madrid:Morata,2001.:Paidós, 22.

12.Álvarez-Gayou, Juan Luis. Cómo hacer investigación cualitativa. Fundamentos y metodología Barcelona, 2005, pp159-163.

13.Van Manem, M. El tacto en la enseñanza. Barcelona: Paidós Educador; 1998.

14.Freire, P. Pedagogía del oprimido. Madrid: Siglo XXI; 1995.

15.Meirieu, F. Frankenstein Educador. Barcelona: Laertes; 1998.

16.Cook, SH., Gordon, FM. Teaching qualitative research: a metaphorical approach. Journal Adv. Nurs. 200447 (6): 649-655.

17.Sopory, P. Metaphor in formative evaluation and message design: an application to relationships and alcohol use. Health Commun. 2005; 17 (2): 149-172.

18.Martín Gordillo, M., Metáforas y simulaciones: alternativas para la didáctica y la enseñanza de las ciencias, Revista Electrónica de Enseñanza de las Ciencias, Vol. 2, No 3, 377-398; 2003.

19.Sontag, S. Las enfermedades y sus metáforas. Madrid: Punto de Lectura; 2003. 